Note

\section{Deficiency of Flagellation in a Bacillus subtilis Pleiotropic Mutant Lacking Transketolase}

\section{Ken-ichi SaSajima and Toshio Kumada}

Biochemistry and Genetics Section, Institute for Fermentation, 17-85, Juso-honmachi 2-chome, Yodogawa-ku, Osaka 532, Japan

Received October 25, 1982

Genetic, biochemical and morphological analysis of flagella formation in gram-negative bacteria, Escherichia coli and Salmonella typhimurium, established an outline of the mechanism of stepwise biogenesis of flagella. ${ }^{1,2)}$ The basal body is joined with the cell surface through several rings. ${ }^{3)}$ In gram-positive bacteria, two ringlike structures also exist. ${ }^{3)}$ The inner ring appears to be in contact with the plasma membrane and the outer ring may be associated with the teichoic acid polymers on the outer surface of the cell. ${ }^{2)}$ Non-flagellate mutants of grampositive bacteria have not been so intensively studied in contrast to gram-negative bacteria. However, Kunst et al., ${ }^{4)}$ Sekiguchi et $a l .{ }^{5)}$ and Ayusawa et al. ${ }^{6)}$ described pleiotropic mutants of Bacillus subtilis, almost all of which were non-motile. All of these mutants seem to be defective in the same unknown component of the cell surface, ${ }^{7)}$ which results in pleiotropic changes such as overproduction of $\alpha$-amylase and exocellular proteases, and inability of flagella formation. The pap mutant, moreover, was not transformable. ${ }^{8)}$

During the course of studies on isolation and characterization of carbohydrate metabolism mutants of Bacillus, it was found that the transketolase mutation $(t k t)$ in $B$. subtilis IFO 12114 generated pleiotropic changes; the transport function of the PEP-dependent D-glucose phosphotransferase system of the tkt mutant, BG2607, was defective, ${ }^{9)}$ and the regulation of synthesis of D-mannitol and sorbitol catabolic enzymes had changed in the mutant strain. ${ }^{10)}$ Further investigation on various functions relating to the cell surface revealed the following facts; (i) cells of the $t k t$ mutant strain formed chains during exponential growth and were thicker than those of the parental strain, (ii) the $t k t$ mutant strain was more sensitive to bacteriophages SP10 and SP01 than the parental strain, (iii) walls of the $t k t$ mutant strain underwent autolysis at a slower rate than did those of the parental strain and (iv) the sporulation frequency of the $t k t$ mutant strain decreased remarkably. ${ }^{11)}$ These pleiotropic properties were generated by a single mutation in the transketolase gene, which was elucidated by the isolation of a true revertant,
BG2694. The true revertant BG2694 was isolated at the same frequency as the forward mutation and showed the same properties as parental strain IFO $12114 .^{9 \sim 11)}$. In this report, another pleiotropic property of $t k t$ mutant BG2607, a deficiency of flagellation, will be described.

The parental strain, B. subtilis IFO 12114, tkt mutant BG2607 and revertant BG2694 were used in this study. The isolation of $t k t$ mutant BG2607 and revertant BG2694 was described previously. ${ }^{9}$

The complete medium ${ }^{11)}$ was used for cultivation of bacteria. To test motility, a loopful of cells of each strain was streaked on semi-solid medium ${ }^{12)}$ with the same composition as the complete medium. Zero point four percent of agar and $0.8 \%$ of gelatin were added into the medium.

To observe flagellation, each strain was cultivated overnight at $37^{\circ} \mathrm{C}$ in $5 \mathrm{ml}$ of the complete medium on a testtube shaker. Cells were stained with flagella-staining reagents. $^{13)}$ Flagella were observed with a Nikon microscope.

During the course of studies on the pleiotropic properties of $t k t$ mutant BG2607, ${ }^{911)}$ it was found that the cells of every culture were non-motile under a phase contrast microscope, while those of the parental strain were certainly motile.

As shown in Fig. 1, tkt mutant BG2607 could not swarm on the semi-solid medium, while parental strain IFO 12114 and revertant BG2694 could swarm.

After staining with reagents for flagella staining, the cells of $t k t$ mutant BG2607 were observed not to have any flagellum, while parental strain IFO 12114 and revertant BG2694 were flagellated (Fig. 2).

As described in previous papers, ${ }^{9 \sim 11)} t k t$ mutant BG2607 was altered in the functions of the cell surface. So the deficiency of flagellation seems also to be related to the

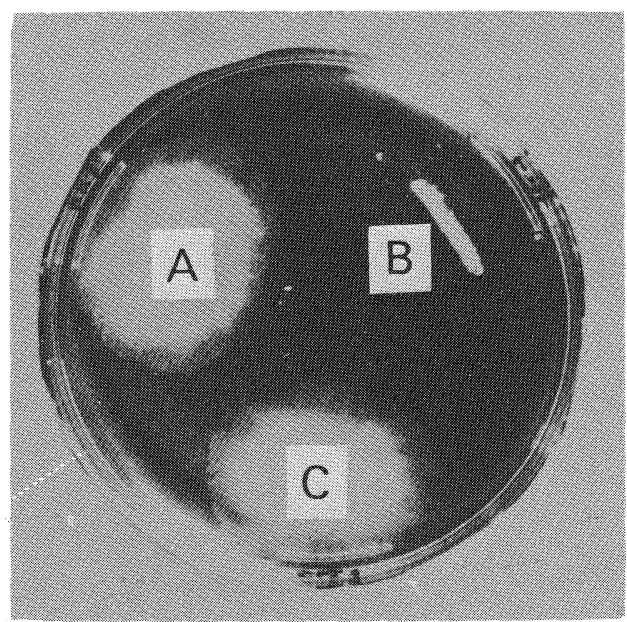

FIG. 1. Motility of Parental Strain B. subtilis IFO 12114, tkt Mutant BG2607 and Revertant BG2694.

A, IFO 12114; B, BG2607; C, BG2694. 

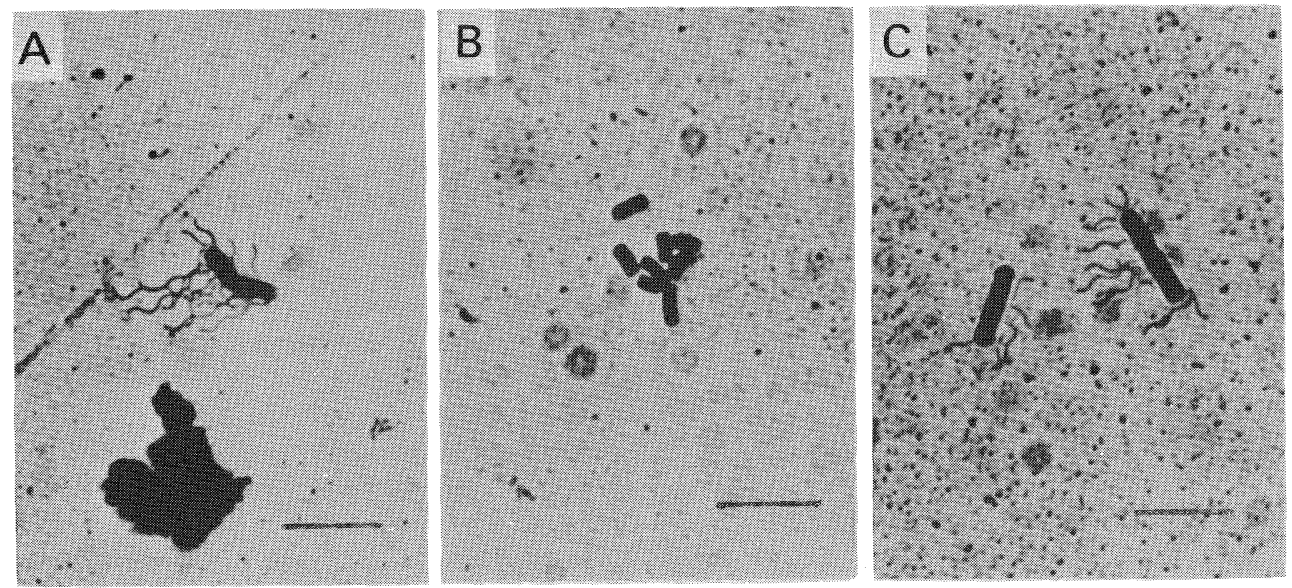

FIG. 2. Flagellation of Parental Strain B. subtilis IFO 12114, tkt Mutant BG2607 and Revertant BG2694.

A, IFO 12114; B, BG2607; C, BG2694. Bars represent $5 \mu \mathrm{m}$.

cell surface change of $t k t$ mutant BG2607 generated by the transketolase mutation. Precise analysis of the cell wall and plasma membrane composition is now in progress.

Non-flagellate mutants of $B$. subtilis, with the various accompanying pleiotropic changes described above, were assumed to be defective as to the cell surface. ${ }^{4,5,7)}$ However, the defective component is unknown in all the mutants.

In gram-negative bacteria, non-flagellate mutants also have been described which are defective in synthesis of lipopolysaccharide. ${ }^{14,15)}$ The core part lipopolysaccharide seems essential for flagella formation in gram-negative bacteria. Non-motile pleiotropic mutants of Caulobacter crescentus have also been described to be defective in pili formation and phage receptors at the same time. ${ }^{16,17)}$

Fein $^{18,19)}$ has reported that autolytic enzymes may be involved in flagellar morphogenesis of B. subtilis 168 and $B$. licheniformis 6346 . The $t k t$ mutant, BG2607, is defective in autolytic activity. ${ }^{11)}$ There is a possibility that the defective autolysis affects the flagellation secondarily.

It is of much interest as to what kind of change has occurred in the cell surface of $t k t$ mutant BG2607 and how the change renders the $t k t$ mutant strain non-flagellate. This is a future problem to be solved.

Acknowledgment. The authors thank Takeda Chemical Industries, Ltd. for financial support of this research.

\section{REFERENCES}

1) T. Iino, Ann. Rev. Genet., 11, 161 (1977).

2) M. Silverman and M. I. Simon, Ann. Rev. Microbiol., 31, 397 (1977).
3) M. L. DePamphilis and J. Adler, J. Bacteriol., 105, 384 (1971).

4) F. Kunst, M. Pascal, J. Lepesant-Kejzlarova, J.-A. Lepesant, A. Biliault and R. Dedonder, Biochimie, 56, 1481 (1974).

5) J. Sekiguchi, N. Takada and H. Okada, J. Bacteriol., 121, 688 (1975).

6) D. Ayusawa, Y. Yoneda, K. Yamane and B. Maruo, J. Bacteriol., 124, 459 (1975).

7) M. Steinmetz, E. Kunst and R. Dedonder, Molec. Gen. Genet., 148, 281 (1976).

8) Y. Yoneda and B. Maruo, J. Bacteriol., 124, 48 (1975).

9) K. Sasajima and T. Kumada, Inst. Ferment. Res. Commun., 9, 17 (1979).

10) K. Sasajima and T. Kumada, Agric. Biol. Chem., 45, 2005 (1981).

11) K. Sasajima and T. Kumada, Inst. Ferment. Res. Commun., 10, 3 (1981).

12) T. M. Joys, J. Bacteriol., 90, 1575 (1965).

13) E. Leifson, J. Bacteriol., 62, 377 (1951).

14) G. F.-L. Ames, E. N. Spudich and H. Nikaido, J. Bacteriol., 117, 406 (1974).

15) Y. Komeda, T. Icho and T. Iino, J. Bacteriol., 129, 908 (1977).

16) A. Fukuda, K. Miyakawa, H. Iida and Y. Okada, Molec. Gen. Genet., 149, 167 (1976).

17) N. Kurn and L. Shapiro, "Current Topics in Cellular Regulation," Vol. 9, ed. by B. L. Horecker and E. R. Stadtman, Academic Press, New York, 1975, p. 41.

18) J. E. Fein and H. J. Rogers, J. Bacteriol., 127, 1427 (1976).

19) J. E. Fein, J. Bacteriol., 137, 933 (1979). 